\title{
DEMOGRAPHIC STUDY OF NASOPHARYNGEAL CARCINOMA IN A HOSPITAL SETTING
}

\author{
M. MUCHIRI
}

\begin{abstract}
Objectives: To highlight demographic pattern of nasopharyngeal carcinoma and determine mode and stage of presentation.

Design: Prospective analytical study.

Setting: Kenyatta National Hospital (KNH)/University of Nairobi, Kenya.

Subjects: One hundred and twenty five patients seen at the ENT department of KNH. These were either referred from other peripheral hospitals or first seen at KNH and satisfied the inclusion criteria. Patients with open neck biopsy were excluded (among other criteria).

Results: Age range of the patients was 13-85 years. Male: Female ratio was 2.2:1 with the highest frequency in the 31-40 years. Most patients presented in late disease (stages III and IV) which carry a poor prognosis. Majority of the patients had neck swelling as the first symptom followed by nose blockage. Several patients referred from peripheral major hospitals and open neck biopsy. Majority of the patients seen were from poor social-economic background.

Conclusion: Nasopharyngeal carcinoma is a common malignancy at KNH. Diagnosis is usually made in late stages of the disease when prognosis after full treatment is poor. There is need to make early diagnosis by heightening the index of suspicion among health professionals and probably health education in the community on the need to seek treatment for persistent neck masses.
\end{abstract}

\section{INTRODUCTION}

Head and neck tumours carry relatively poor prognosis in our setup (1-3). Their late presentation come in a physically horrifying and dramatic picture of presentation to the patient, health workers, and relatives since they affect the very important vegetative functions of feeding and breathing besides being situated on the very exposed parts of the body.

Nasopharyngeal carcinoma (NPC) has very diverse picture of presentation and no characteristic macroscopic, features. Its symptoms depend on local-regional and distant spread. Globally, the highest incidence of NPC is found in Mongolian races of southern China, Hong Kong and Southern
East Asia Chinese. In Africa, Kenya, Tunisia and Algeria have higher incidences than non-Mongolian races (4-6).

Since the last decade there have been major advances in technology and patient care. CT scan, rigid nasoendoscopy and tissue biopsy that improve staging are now routinely performed. The system of staging the disease has since been standardised globally from American Joint Committee on Cancer (AJCC) to Union Internationale le Cancer (UICC), which has been edited through the years. The 1997 (5th) edition is the one in use at ENT Department - KNH.

Most of NPC arise from the Fossa of Rosenmuller on the lateral wall of the nasopharynx just behind the tubal elevation $(2,4,7)$. This fossa opens into 
the nasopharynx at a point just below the foramen lacerum. Lateral to the fossa are the carotid canal opening, foramina of ovale and spinosum, mandibular nerve and prestyloid compartment of the parapharyngeal space $(3,4,7-10)$.

This study highlights important signs and symptoms of nasopharyngeal carcinoma some of which are overlooked and often not sought for by the clinician. The study also compares various demographic variables of the disease noted locally with findings in other studies.

\section{MATERIALS AND METHODS}

Patients included in the study were those seen at ENT department with signs and symptoms of NPC. A thorough history of illness and meticulous physical examination were done by the author and findings entered in a questionnaire. Patients with neck masses were sent for fine needle aspirate biopsy (FNAB) and base of skull/neck CT scan. Rigid nasal endoscopy then followed usually under local anaesthesia where a tissue biopsy was taken for histology. Disease staging was then done using the UICC 5th edition classification. Patients in poor general conditions like severe odynodysphagia and dehydration were admitted for supportive therapy and investigations. After staging, patients were booked to tumour board attended by ENT consultants, radiologists, oncologists and registrars in all above disciplines. This board sits on Friday morning at ENT department $\mathrm{KNH}$, and deliberates on best treatment option for each patient presented.
All information gathered was entered in a questionnaire and verified by the author. Analysis was done using the specific statistical package software version 11 .

\section{RESULTS}

During the eight months of data collection 125 subjects fitted the inclusion criteria. Age range of the patients was 13 to 85 years with the highest number of patients in the 31 to 40 years age range $(22.4 \%)$. The youngest patient was 13 years while the oldest was 85 years old. Male: female ratio was 2.3:1.

Table 1

Age frequency distribution

\begin{tabular}{lcc}
\hline Age rage (years) & No. & $(\%)$ \\
\hline $11-20$ & 14 & 11.2 \\
$21-30$ & 13 & 10.4 \\
$31-40$ & 28 & 22.4 \\
$41-50$ & 26 & 20.8 \\
$51-60$ & 25 & 20.0 \\
$61-70$ & 11 & 8.8 \\
$71-80$ & 5 & 4.0 \\
$81-90$ & 3 & 2.4 \\
\hline Total & 125 & 100 \\
\hline
\end{tabular}

Figure 1

Age distribution of the 125 patients

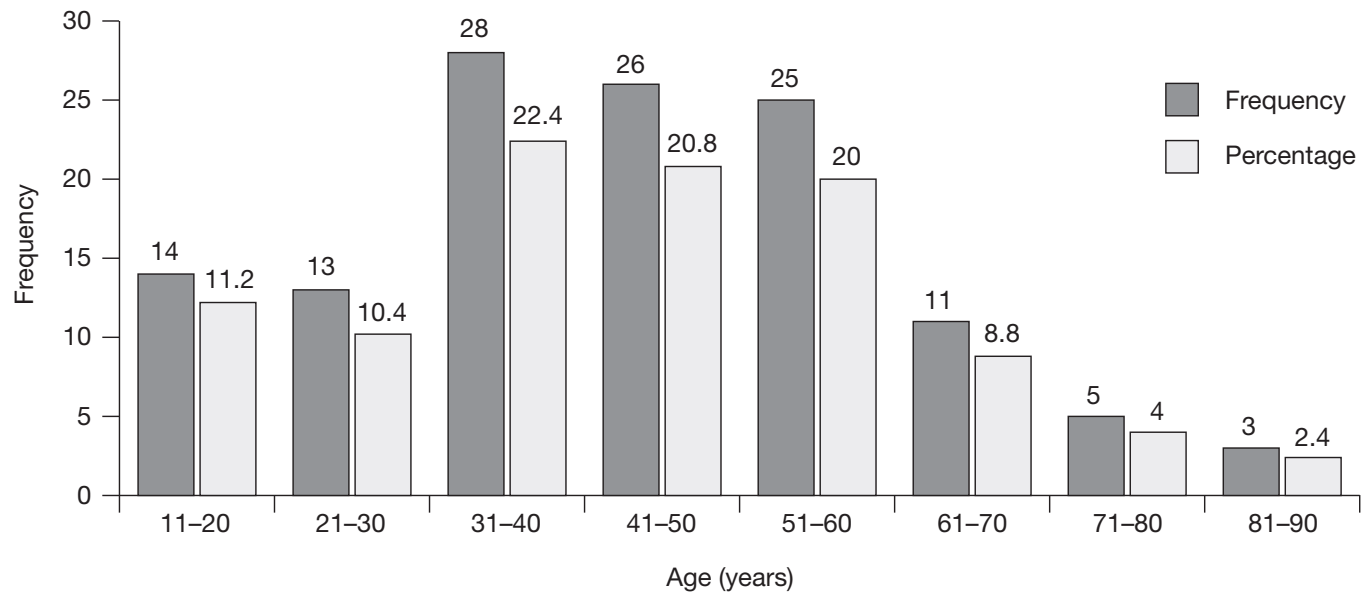

Thirty two per cent of the patients recorded use of tobacco. One chewed while one sniffed. It was not possible to quantify these latter cases in pack years. 
Table 2

Smoking habits

\begin{tabular}{lcc}
\hline Pack years & No. & $(\%)$ \\
\hline $1-10$ & 29 & 23.2 \\
$11-20$ & 8 & 6.4 \\
Over 20 & 4 & 3.2 \\
Total smokers & 41 & 32.8 \\
Total non-smokers & 84 & 67.2 \\
\hline Total & 125 & 100 \\
\hline
\end{tabular}

1 pack years $=$ No. of cigarettes smoked per day times years smoked divided by 20

Forty one per cent gave history of alcohol use, duration varying between two years to 60 years and amount ranging between 104 alcohol units to 2,848 units per annum.

( 1 unit of alcohol $=1 / 2$ pints of beer $=1$ measure of whiskey $=$ glass of wine)

Fifty six per cent of patients were peasant farmers in rural Kenya.

Patients were grouped into regions (Figure 2). Patients from Meru were grouped together with those from central Kenya as reflected in their similarities with respect to geographic/ climatic and socio-cultural practice (Mt. Kenya region). Similarly, those from Eastern and North Eastern were grouped together. Majority of patients were seen to come from Mt. Kenya/Central region (38.4\%) while the least came from coast province $(40 \%)$.

Majority of patients in Eastern/North Eastern group came from Ukambani districts. Generally most patients were noticed to come from the highland areas of Mt. Kenya region, Rift Valley highlands of Kericho and Western Kenya highlands. This compared well with the retrospective study done in 1985 for patients seen between 1973 and 1983 by Mwangi. He found that majority of patients were of Kikuyu tribe and the least affected tribe to be Mijikendas (Coast) (2).

The environmental conditions of the people of southern China differ markedly with the Kenyans. It is likely that whereas an ingested factor may be carcinogenic in China and the West, this factor/ trigger may be environmental in our set-up. EBV is the likely culprit in Kenya (1). A conclusive study needs to be done to try and resolve this possibility.

Symptoms: The most common symptoms were neck swelling (85\%), unilateral nasal blockage $71 \%$, epistaxis $44.8 \%$ and unilateral hearing loss $36.6 \%$. Most patients had a multiplicity of symptoms. Many patients (48\%) had symptoms for between 4-7 months before seeking any medical attention. (Range of duration of symptoms was 1-39mo).

Figure 2

Region/province of residence

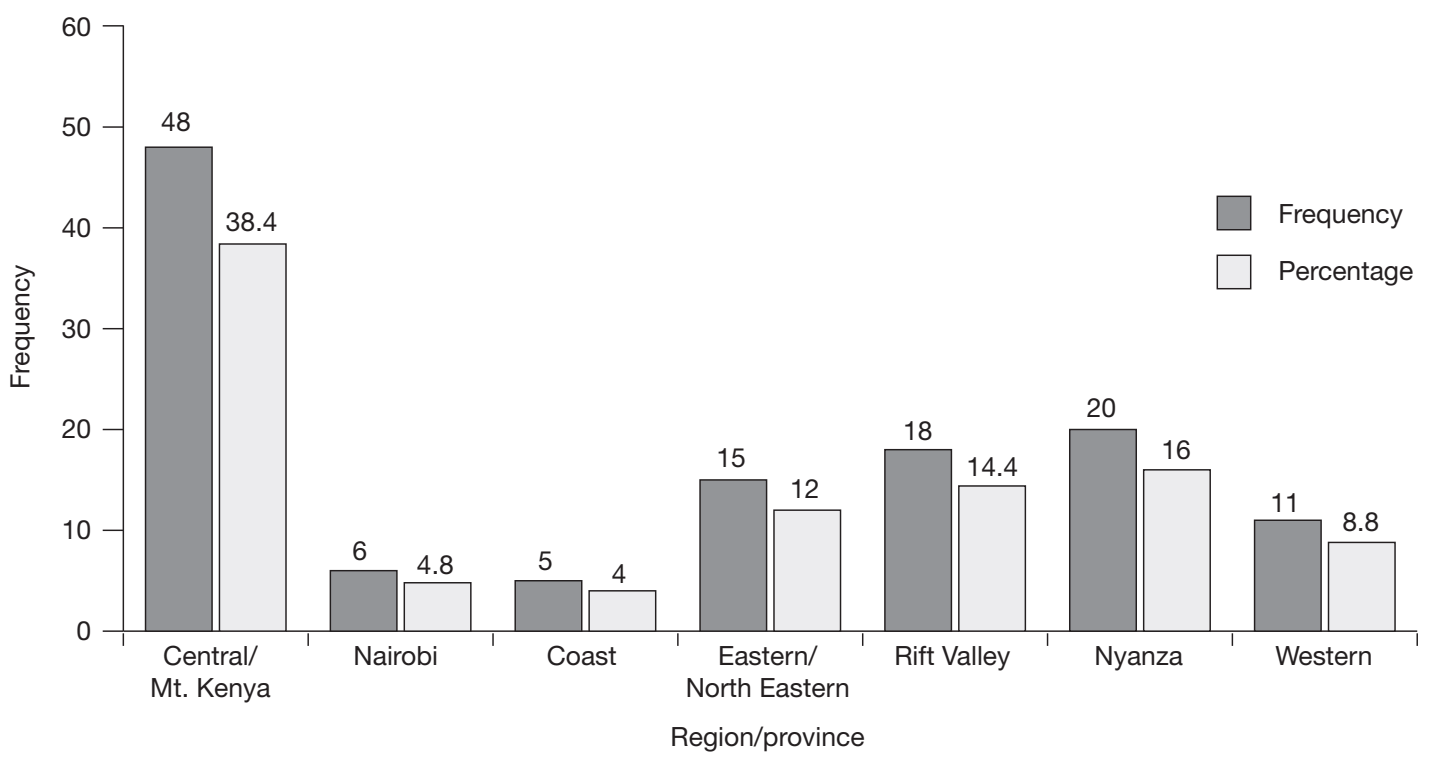


Table 3

Presenting symptoms

\begin{tabular}{llllll}
\hline Symptom & Frequency & $(\%)$ & Symptom & Frequency & $(\%)$ \\
\hline Neck swelling & 100 & 80.0 & Dysphonia & 7 & 5.6 \\
Nasal blockage & 89 & 71.2 & Dysphagia & 18 & 14.4 \\
Epistaxis & 56 & 44.8 & Trismus & 26 & 20.8 \\
Nasal discharge & 1 & 0.8 & Proptosis & 13 & 10.4 \\
Nasal growth & 13 & 10.4 & Diplopia & 8 & 6.4 \\
Ear blockage & 25 & 20 & Failing vision & 8 & 6.4 \\
Otalgia/ear pain & 11 & 8.8 & Facial numbness & 14 & 11.2 \\
Tinnitus & 17 & 13.6 & Others & 24 & 19.2 \\
Hearing loss & 47 & 37.6 & Total & 125 & 100 \\
\hline
\end{tabular}

Figure 3

Cranial nerve involvement

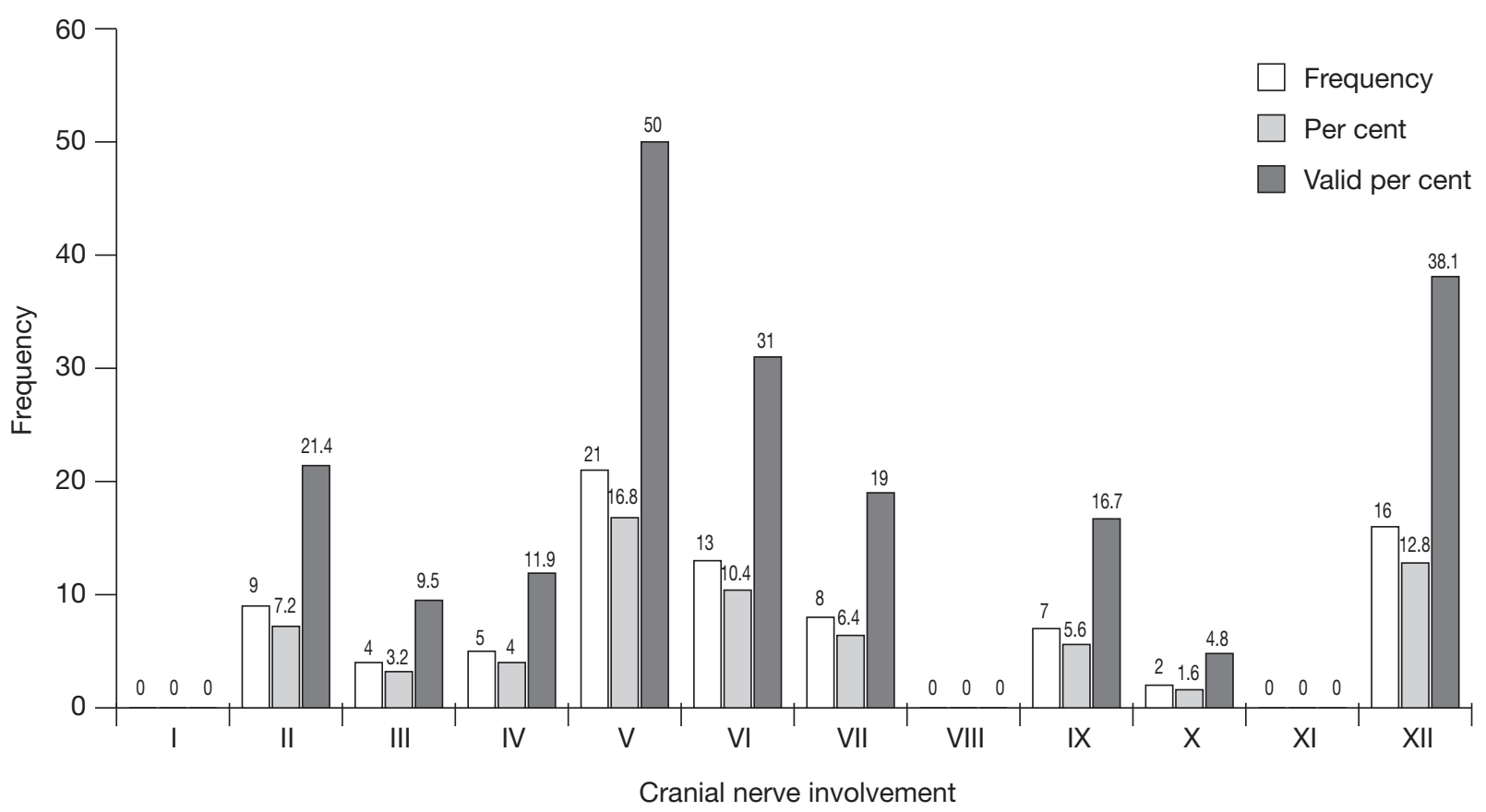

CT scan revealed $26.2 \%$ of the patients had evidence of bone erosion while $13.8 \%$ had intracranial involvement.

Eighty eight per cent of the patients had cervical node involvement mainly involving levels II and III. Most of these were N3a. (One patient had N3b).

Conductive hearing loss (CHL) was noted in $36 \%$ of patients out of whom $22.2 \%$ also had otitis media with effusion (OME).

A total of 42 patients had cranial nerve $(\mathrm{CN})$ involvement most of who had multiple nerves involved. The most involved $\mathrm{CN}$ was trigeminal nerve. Its involvement came in various presentations like feeling of crawling movements under the skin, hotness, numbness, "feels different" over the area of the nerve distribution on the face. Other cranial nerves involved were XII, II, VI, XI among others.

Staging: Only 11 patients presented in early stage disease (stages I and II). Sixty three per cent of the patients presented in stage IV while $26 \%$ came in stage III disease. 


\section{DISCUSSION}

In 1948, Clark et al found no nasopharyngeal cancer in Kenya. In 1965, Clifford et al showed that $30 \%$ of all head and neck cancers are NPC (which constituted 9\% of all cancers). In 1971, Wen et al showed that NPC constituted 0.48 per $1,000,000$ population in Kenya.

In 1993, American Society for Radiology and Oncology (ASTRO) refresher course on NPC highlighted Kenya as an area of high incidence. These studies reveal an inconsistency and hence there is need to perform a large cohort standardised study on NPC in the region. The aetiology of NPC has been assigned genetic and environment factors some of them are;

(i) Genetic predisposition duly presence of HLA BW 46, A2 and B17 and haplotype A2-B W46 and AW19-B17 have a higher risk of getting $\operatorname{NPC}(4,7,11)$.

(ii) Mutated suppressor gene P53 is related to cell progression and malignancy. An association has been seen to exist between NPC and P53 codon 72 polymorphism. The P53 proline (pro) homozygotes are related to a lower risk of NPC development $(4,7,11-13)$. It would be important to undertake local studies to determine the local genetic risk factors.

(iii) Epstein bar virus (EBV) primary infection takes place in childhood and is always accompanied by seroconversion and harboring of virus in dormant state for life $(4,7,8,12)$. These viruses-may be reactivated resulting in raised serological titres in immune suppressed states. Important EBV related Abs in NPC are;
(a) IgA and IgG to viral capsid antigen (VCA)
(b) IgA and IgG to early EBV antigen (EA)
(c) Antibody to nuclear EBV antigen (EBNA)
(d) Antibody dependent cell cytotoxicity (ADCC) $(4,7,8)$.

These antibodies have been used to follow up patient response to treatment. IgA/VCA, IgG/ VCA, IgG/EA titre are related to tumour load. An upsurge of VCA, EA and NA antibodies indicate clinical recurrence and or metastasis.

ADCC antibody titre determines survival. The higher the titre the better the prognosis. These antibodies titres are not routinely done at $\mathrm{KNH}$. Availability of such titres would significantly improve our understanding and management of NPC.

NPC is malignant squamous cell carcinoma which frequently arises from the lateral wall of the nasopharynx at the fossa of Rosenmuller and metastasis to nodes occurs early courtesy of the rich blood supply of the region $(4,7,8)$.

This may partly explain why most patients present in late disease as the tumour grows locally and spreads to the cervical nodes and other local sites like base of skull hence $\mathrm{CN}$ involvement and laterally towards the Eustachian tube to cause otology symptoms (14). Most patients being in the rural areas and busy peasant farmers shrug off mild painless neck swellings, occasional nasal blockage and at times mild epistaxis as "insignificant". The triad of CHL, elevation/immobility of ipsilateral soft palate and pain over same side of head (CNV involvement) represent symptoms of local invasion (Trotters triad). A thorough eye, ear, nose, throat ENT examination must include full neurological examination of face, oral cavity and neck. FNAB must be done for persistent neck masses.

A rigid nasal endoscopy is mandatory for suspected NPC at which time a tissue biopsy is also taken for histoglogy. This is-advised to be done after the CT scan since post biopsy inflammatory process/trauma could appear like growth where there were none and distort the normal image of the nasopharynx (15). CT scan provides information vital for disease staging.

Locoregional spread, cervical node involvement, base of skull erosion (especially around the foramina) and intracranial extension can be assessed. $(4,7,15,16)$. Unfortunately in our setup many patients cannot afford a CT scan. Some bring the results many months after the request for the $\mathrm{CT}$ scan was made and hence stage of disease further advanced. In this study, $26.6 \%$ of patients had evidence of skull bone erosion while 13.8\% had evidence of intracranial extension on CT scan. Both are poor prognostic factors. Eighty seven point two per cent of the study patients had anaplastic undifferentiated squamous cell carcinoma. Thiscorrelates well with studies done earlier $(1,2,17)$.

Late stage NPC (Stages III and IV) carry a poor prognosis with a one year survival rates of 30-35\% $(1,18)$. Only eight patients were in stage II and three in stage I. The later has a good prognosis or $87-90 \%$ one year survival rates (18). 
During the course of this study I encountered many patients who had been sent to $\mathrm{KNH}$ radiotherapy department with diagnosis of NPC made from $\mathrm{CT}$ scan and open neck biopsy already done at either district or provincial hospital most often by a general surgeon or general practitioner.

Any incision over the neck for biopsy iatrogenically pushes disease stage to IV thus worsens prognosis.

The following are recommended;

(i) The benefit of early diagnosis, investigations work up and treatment for NPC cannot be over emphasised.

(ii) Any patient with persistent neck swelling should be advised to have a FNAB. Suspicion is further heightened if there are other ipsilateral face, ear, nose or eye related symptom (19).

(iii) Open neck biopsy should never be done unless exhaustive investigations have been done to rule out head and neck tumours. These must include oropharyngeal panendoscopy and biopsies in areas known to hide malignancy-coffins corner, nasopharynx, tonsilar poles, retromolar trigone and piriform sinus.

(iv) Teaching of paramedics and medics should also emphasise these facts.

(v) Further studies e.g. histochemical and gene mapping studies to characterise NPC in our region would help understand the disease further and work towards identifying a screening test (20).

\section{ACKNOWLEDGEMENTS}

Special thanks and gratitude to Dr. P. Mugwe who supervised me and staff of Kenyatta National Hospital who assisted me in this study.

\section{REFERENCES}

1. Oburra, H.O. Late presentation of laryngeal and nasopharyngeal cancer in KNH. East Afr. Med. J. 1998; 75: 223-226.

2. Mwangi, J.C. Nasopharyngeal carcinoma seen at KNH between 1973-1983 Dissertation, in part fulfilment for MMed Surgery, 1985.

3. Feinmesser, R., Miyazaki, I., Cheung, R., et al. Polymerase chain reaction in diagnosis of NPC. New Engl. J. Med. 1992; 326: 17-21.
4. John, W., Mark, G. and Janet, W. Nasopharyngeal carcinoma. Head and Neck Surgery (Stell and Maran) $4^{\text {th }}$ edn. Butterworth-Heinmann Ltd. 1994; 397-407.

5. Neel, H.B. NPC clinical presentation, diagnosis and treatment. Otolaryngologic Clinics of North America. 1985; 18: 479-488.

6. Tai, T.H.P. and Mould, R.F. Descriptive epidemiology of NPC. Current Oncol. 2001; 2: 2-68.

7. Chew, C.T. Nasopharynx In: Scotts Browns; Otolaryngology $4,5^{\text {th }}$ edn. Ian S.M. Bull. T.R. Butterworth and Co. Ltd. 1987; 315-330.

8. Rynd, M. Tropical and geographical medicine. Nasopharyngeal Cancer Review. 1993; 43: 59-65.

9. Gray, H. The pharynx. Descriptive and surgical anatomy. Paragon. 563.

10. Chaurasias, B.D. Nasopharynx. Human anatomy, Regional and applied, $2^{\text {nd }}$ edn. Satish Kumar Jain. 197.

11. Tsai Ming-H, Lin, C.D. and Hsieh, Y.Y. Prognostic significance of P53 codon 72 ploymorphism in NPC. Laryngoscope United States 2002; 112: 116-119.

12. Larry, H. and William, N.H. Archives of otolaryngology. Head and Neck surgery. 1963; 109: 143-147.

13. Mow-Ming, H., Jeng-Yuh, K. and Yen-Lang, C. Elevated levels of 1L2 receptor and tumour necrosis factor in NPC. Archives of otolaryngology. Head and Neck Surg. 1991; 117: 1257-1259.

14. Negan, R.K.C., Yiu, H.H.Y., Cheng, H.K.M., et al. Central nervous system metastasis from NPC. Cancer (United States), 2002; 94: 398-405.

15. Chong, V.T., Fan, Y.T. and Khoo, J.B. Computed tomography and MRI findings in paranasal sinuses involved with NPC. Ann. Med. Singapore. 1998; 27: 800-804.

16. Kao, C.H. and Ho, Y.J. Comparison of positron emission tomography in detection of lymph node metastasis of NPC. Ann. Ontol. Rhinol. Laryngol. (United States). 2000; 109: 1130-1134.

17. Kwong, D.L., Nicholas, J., Wei, W.I., et al. Correlation of endoscopic and histologic findings- before and after treatment for NPC. Head and Neck (United States). 2001; 23: 34-41.

18. Ho-Sheng, L. Malignant nasopharyngeal tumours. Wayne State University/Detroit Medical Center. e-medicine.com.Inc. 2002.

19. Teo, P., Leung, S.F., Yu, P., Lee, W.Y., et al. A retrospective comparison between different stage classifications for NPC. Brit. J. Radiol. 1991; 64: 901-908.

20. Pak, N.W., To, K.F. and van Hessel, G.A. In vivo diagnosis of NPC using contact rhinoscopy. Laryngoscope (United States). 2001; III(8): 1453-1458. 